\title{
Paradoxical Stimulation of Matrix Metalloproteinase-9 Expression in HT 1080 Cells by a Broad-Spectrum Hydroxamate-Based Matrix Metalloproteinase Inhibitor
}

\author{
E. MAQUOI, ${ }^{\mathrm{a}}$ C. MUNAUT, ${ }^{\mathrm{a}}$ A. COLIGE,${ }^{\mathrm{b}}$ C. LAMBERT, ${ }^{\mathrm{b}}$ F. FRANKENNE, ${ }^{\mathrm{a}}$ A. NOEL,${ }^{\mathrm{a}}$ F. GRAMS, ${ }^{\mathrm{C}}$ H.-W. \\ KRELL, ${ }^{d}$ AND J.-M. FOIDART ${ }^{\mathrm{a}}$ \\ ${ }^{a}$ Laboratoire de Biologie des Tumeurs et du Développement, Université de Liège, Tour de Pathologie (B23), Sart Tilman, B-4000 Liège, \\ Belgium \\ ${ }^{\mathrm{b}}$ Laboratoire de Biologie des Tissus Conjonctifs, Université de Liège, Tour de Pathologie (B23), Sart Tilman B-4000 Liège, Belgium \\ Boehringer Mannheim, GmbH, Mannheim, Germany \\ ${ }^{d}$ Boehringer Mannheim, GmbH, Penzberg, Germany
}

\section{INTRODUCTION}

Due to their unusual ability to cleave and degrade a variety of ECM components, including triple-helical type IV collagen, two members of the family of matrix metalloproteinases (MMPs), MMP-2 and MMP-9, are thought to play critical roles during tumor invasion and metastasis. The proteolytic activity of mature MMPs is modulated by a group of specific inhibitors, the tissue inhibitors of metalloproteinases (TIMPs). Excessive ECM degradation observed during tumor invasion and metastasis frequently correlates with an excess of active MMPs. Therefore, adding exogenous inhibitors was contemplated for anticancer therapy. Indeed, synthetic MMP inhibitors have been demonstrated to reduce tumor invasion and metastasis in several in vitro and in vivo models. ${ }^{1}$

Here, we show that some of these synthetic inhibitors paradoxically enhance the production of MMP-9 even as they inhibit the activity of others (MMP-2, MT1-MMP).

\section{RESULTS}

\section{GI Reduced the Invasion of HT1080 Cells through Type IV Collagen}

We first demonstrated, using Transwell chambers coated with type IV collagen, ${ }^{2}$ that GI129471 (GI, $\left.1 \mu \mathrm{M}\right)$, an hydroxamate-based inhibitor, ${ }^{3}$ reduces HT1080 cell invasion up to about $20 \%$ of that of the vehicle-treated cells (data not shown). In contrast, neither the ability of the cells to migrate through uncoated filters nor their metabolism were affected by GI, indicating that the inhibition of HT1080 invasion was most probably mediated through reduced proteolytic activity. Gelatin zymography of conditioned media from the chemoinvasion assays revealed that HT1080 essentially secreted pro-MMP-2 and pro-MMP-9. In addition, two activated forms of MMP-2 and a 120-kDa gelatinase activity (corresponding to a complex between TIMP-1 and the pro-MMP-9) were also detected (FIG. 1). Upon treatment with GI, the MMP-2 activated forms were undetectable, indicating the inhibition of MT1-MMP activity. Unexpectedly, the levels of both pro-MMP-9 and 120-kDa complex were strongly increased.

\section{GI Stimulated MMP-9 Production by HT1080 Cells}

To further investigate the effect of GI on MMP-9 secretion, HT 1080 cells were treated for 48 hours with graded GI concentrations $(0-1 \mu \mathrm{M})$. GI dose-dependently stimulated the secretion of pro-MMP-9 with a maximal 6.6fold stimulation at $1 \mu \mathrm{M}$ (FIG. 1). In contrast, pro-MMP-2 processing was dose-dependently inhibited. On the other hand, neither rTIMP-1 nor rTIMP-2 $(0.01-1 \mu \mathrm{M})$, the physiological MMP inhibitors, significantly modulated MMP-9 secretion (data not shown).

Using quantitative RT-PCR, we have shown that GI $(0-1 \mu \mathrm{M})$ treatment increases the MMP-9 mRNA level (FIG. 2 ). The effect of GI was dose dependent up to the concentration of $1 \mu \mathrm{M}$. Northern blot analysis showed that GI does not alter MMP- 1, MMP-2, MT1-MMP, TIMP-1, or TIMP-2 mRNA levels (data not shown). 
FIGURE 1: GI modulates MMP-9 secretion in HT1080 cells. Conditioned media obtained from cells cultured for $48 \mathrm{hr}$ with GI (0-1 $\mu \mathrm{M})$ were analyzed by gelatin zymography. The relative gelatinolytic activity corresponding to MMP-9 was evaluated by scanning densitometry. A value of 1 was arbitrarily given to the control condition. Relative stimulation (fold) of MMP-9 secretion is shown.

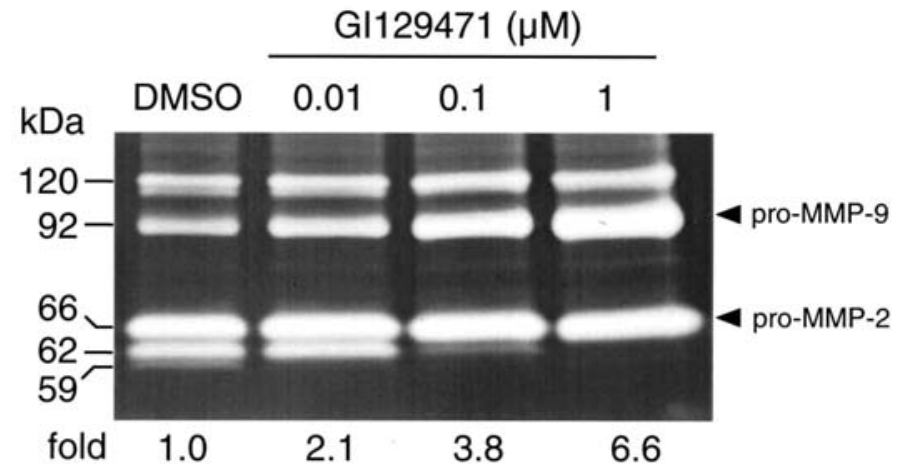

FIGURE 2: GI increases MMP-9 mRNA levels in HT1080 cells. MMP-9 mRNA levels were quantified by quantitative RT-PCR and densitometric analysis of autoradiographies of the gels. All results were corrected by densitometric data obtained for the 28S rRNA. A value of 1 was arbitrarily attributed to the relative level of MMP-9 mRNA measured in control condition.

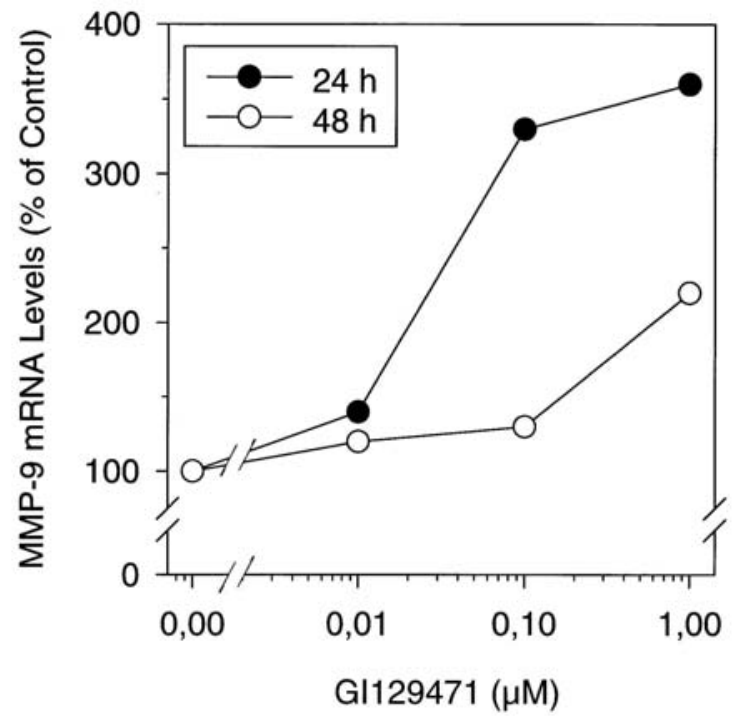

\section{GI Stimules the Transcriptional Activity of the MMP-9 Promoter}

In order to assess the effect of GI on MMP-9 transcription, HT1080 cells were transiently transfected with pNA7.8 reporter vector. This construct was made up of the murine MMP-9 promoter $(-7745$ to $+1 \mathrm{bp})$ linked to the $\beta$-galactosidase reporter gene. In the presence of GI, a fourfold stimulation of $\beta$-galactosidase activity was noted after 48 hours (data not shown). These data clearly demonstrated that the GI-stimulated MMP-9 synthesis was mediated through an enhanced transcription of the MMP-9 gene.

\section{CONCLUSIONS}

Our findings clearly demonstrate that GI129471, a broad-spectrum hydroxamate-based MMP inhibitor, could, in addition to its classical inhibitory activities, specifically upregulate both transcription and synthesis of proMMP-9. Our data thus emphasize the need to carefully investigate the potential side effects of such unspecific broad-spectrum MMP inhibitors. 
Published in: Annals of the New York Academy of Sciences (1999), vol. 878, pp. 744-746 Status: Postprint (Author's version)

\section{REFERENCES}

1. TALBOT, D.C. \& P.D. BROWN. 1996. Experimental and clinical studies on the use of matrix metalloproteinase inhibitors for the treatment of cancer. Eur. J. Cancer, 14: 2528-2533.

2. Maquoi, E., A. NoÉl, F. Frankenne, H. Angliker, G. Murphy \& J.M. FoidArT. 1998. Inhibition of matrix metalloproteinase 2 maturation and HT1080 invasiveness by a synthetic furin inhibitor. FEBS Lett. 424: 262-266.

3. CAMPION, C, J.P. DiCKENS \& M.J. CRIMMIN. PCT Patent Application, WO 90/05719, 1990. 